\title{
From Sensors to Standards: How NIST on a Chip is Transforming International Metrology
}

\author{
Barbara Goldstein ${ }^{1}$ \\ ${ }^{1}$ National Institute of Standards and Technology, Physical Measurement Laboratory, 100 Bureau \\ Drive, Gaithersburg, MD 20899, barbara.goldstein@nist.gov
}

\begin{abstract}
Summary:
What draws the line between a sensor and a standard? If precision measurement devices can be reduced to the size of a grain of rice, embedded directly in products, deployed on submarines or satellites without the need for periodic calibration, how does the international metrology framework need to change to adapt? And what commercial applications does it enable? These are the very questions raised by the successes of the NIST on a Chip program, which is transforming how precision measurements are being brought out of the lab and deployed at point of use through a suite of miniaturized, fit-for-purpose, quantum-based traceable sensors.
\end{abstract}

Keywords: Quantum Revolution, Metrology, Redefinition of the SI, Industry 4.0, Technology Readiness, Quantum Sensing

\begin{abstract}
NIST on a Chip Vision
In the 1990s, when the world's best and only traceable measurements were made in laboratories filled with complex equipment that required highly skilled staff to operate, NIST researchers, together with U.S Defense Advanced Research Projects Agency (DARPA), had the bold notion that it might be possible to shrink an atomic clock down to a low cost, lowpower chip-scale device. The successes arising from these early and sustained investments and industry collaborations ${ }^{1}$ not only led to commercially available chip-scale atomic clocks, but motivated an entire research venture into developing chip-scale standards.
\end{abstract}

The NIST on a Chip program ${ }^{2}$, or NOAC, is revolutionizing metrology by making precision measurements available at point-of-use through a suite of intrinsically accurate, quantum-based devices intended to be deployed nearly anywhere and anytime, performing uninterrupted without the need for NIST's traditional measurement services.

These quantum-based measurement technologies will enable users to make precision measurements referenced to the International System of Units (SI) wherever they're most needed - on factory floors, on satellites, in hospital diagnostic centers, in research labs, and ultimately in homes, automobiles, personal electronic

\footnotetext{
${ }^{1}$ https://www.nist.gov/noac/success-story-chipscale-atomic-clock

${ }^{2}$ https://www.nist.gov/noac
}

devices, and more. NOAC is helping to "democratize" measurement technology, by drastically reducing the cost and increasing the availability of precision measurements that could previously only be delivered at the world's best metrology institutes.

The suite of ultra-compact, inexpensive, lowpower devices being developed within the program can measure the full range of quantities including time and frequency, distance, mass and force, temperature and pressure, electrical and magnetic fields, current and voltage, and fluid volume and flow.

The program envisions eventually combining multiple measurement capa-

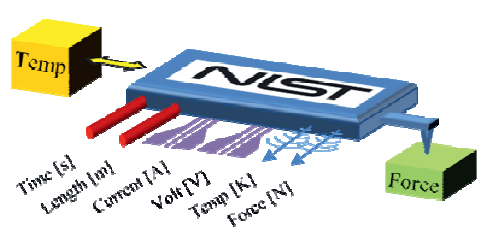
bilities onto an integrated platform to enable, for example, a single, embeddable chip that senses absolute temperature, pressure, and humidity to immediately detect any excursions in safe storage conditions of sensitive goods, such as vaccines or food. Other applications will leverage inexpensive mass fabrication, leading to applications such as a chip-scale radiation monitor that could be embedded in every driver's license or other ID card to serve as a ubiquitous monitor or early-warning system for radiation exposure.

These NIST-pioneered technologies will be manufactured and distributed by the private 
sector, opening new technology transfer and lab-to-market opportunities in accordance with NIST's goal of strengthening U.S. economic competitiveness by supporting advanced manufacturing.

\section{Defining Criteria for NOAC Devices}

The quantum-based standards and sensors being developed within the NOAC program are designed to be:

Deployable to where customers need them, such as on the factory floor, embedded into products, in a laboratory environment, in space or at home.

Flexible, providing a broad range of "zero chain" SI-traceable measurements and standards that are configurable into a single small-form package and adaptable to customers' requirements.

Manufacturable, with production costs that scale appropriately for applications, such as low-cost/high-volume for broad deployment.

Reliable, providing either the right value of a measurement or no value at all.

Fit-to-Function, tending towards small size, low power consumption, rugged, easily integrated and operated, with an operating range and uncertainty required by the application.

\section{Propitious Timing}

NOAC at the Convergence of Industry Trends NGT

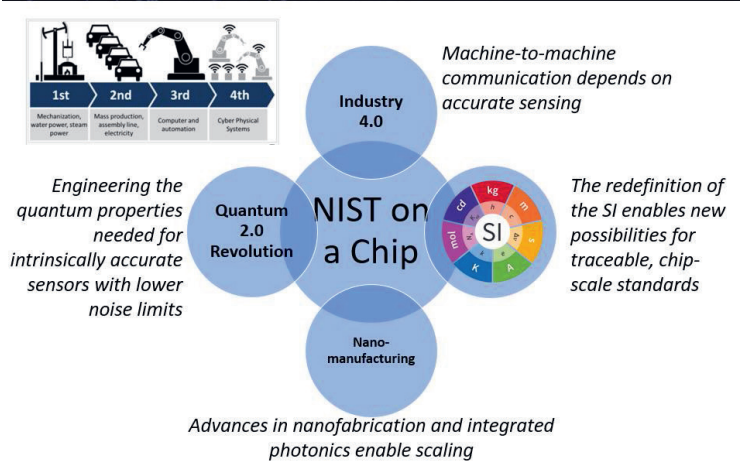

NOAC innovations will be increasingly valuable to industry, medicine, defense, and science because of the current convergence of major trends in technology advancement. For example, Industry 4.0 is an optimization strategy in which the machinery of industrial production no longer simply "processes" the product, but the product communicates with that machinery in a digital choreography of production.

This approach will come to redefine the consumer-manufacturer relationship, as products in the field (i.e., in the Internet of Things) communicate back to the manufacturing ecosystem that produced them (the Industrial Internet of
Things) to influence everything from nextgeneration product design, supply chain management, peer-to-peer consumer networking, product maintenance and end-of-life. This new paradigm won't be possible without accurate sensors both in the field and in the plant to provide reliable information to drive automated, machine-to-machine communication and decision making.

At the same time, the emergence of the second quantum revolution - which depends on the control and manipulation of matter at the most fundamental levels - will spur a new generation of technologies based on phenomena such as entanglement and superposition. The preservation and manipulation of these very fragile quantum states will require reliable, in-situ sensors and measurements, a NOAC goal. In addition, advances in quantum information science will enable unprecedented advances in measurement precision and thus fuel a new generation of quantum-accurate standards and measurements.

Finally, the explosive demand for high-speed transfer of ever-larger volumes of data will benefit directly from NOAC's pioneering work in miniaturized photonic channels, novel signal transduction schemes, and accurate calibration standards for devices that must operate at unprecedented frequencies.

\section{Opportunities for Partnerships}

The NIST on a Chip program was built through collaboration. NOAC technologies are at varying stages of technology readiness. NIST is actively building partnerships with industry, both domestically and globally, to bring these innovations from lab to market. A portfolio of patents available for licensing are available at https://www.nist.gov/noac/patents. Potential partners are encouraged to explore opportunities for engagement. 